\title{
Beneficial Effects of Drossinakis Bio-influence (With Infrared Thermal and Electromagnetic Fields) on the Development of Experimental Graffi Myeloid Tumors in Hamsters. Hematological Studies
}

Reneta Toshkova $^{1} \quad$ Ignat Ignatov $^{2 *} \quad$ Elissaveta Zvetkova $^{3} \quad$ Georgi Gluhchev $^{4} \quad$ Christos Drossinakis $^{5}$

1. $\mathrm{PhD}, \mathrm{MD}$, Professor, Institute of Experimental Morphology, Pathology and Anthropology with Museum, Bulgarian Academy of Science (BAS), Acad. G. Bonchev Street, bl. 25, Sofia 1113, Bulgaria

2. DSc, Professor, Scientific Research Center of Medical Biophysics (SRCMB),

N. Kopernik Street, 32, Sofia 1111, Bulgaria

3. $\mathrm{PhD}, \mathrm{MD}$, Assoc. Prof., Bulgarian Biorh

eological Society, Bulgarian Academy of Science (BAS),

Acad. G. Bonchev Street, bl. 4, Sofia 1113, Bulgaria

4. $\mathrm{PhD}$, Assoc. Professor; Institute of Information and Communication Technologies, Bulgarian Academy of Science (BAS), Acad. G. Bonchev Street, bl. 2, Sofia 1113, Bulgaria

5. Dr. h.c, Professor, IAWG - INTERNATIONALE Akademie für Wissenschaftliche

Geistheilung Königsteiner Str. 61 a, 65929 Frankfurt Höchst, Germany

\begin{abstract}
The aim_of the present experiments was to study the effect of bioinfluence - Infrared thermal field (ITF) and electromagnetic fields (e.m. fields) on the progression of experimental tumor - Graffi myeloid tumor in hamsters Spectral analysis methods called Non-equilibrium spectrum (NES) and Differential non-equilibrium spectrum (DNES) for $1 \%$ solutions of blood serum from hamsters with cancer and healthy hamsters were investigated. Drossinakis has a beneficial effect on the Graffi tumor-bearing hamsters. Results are obtained with the DNES method - compared to the control serum samples of hamsters with cancer and healthy hamsters. The spectrum was obtained in the range $\left(-0.0937 \mathrm{eV} ; 13.23 \mu \mathrm{m} ; 755 \mathrm{~cm}^{-1}\right)-\left(-0.1387 \mathrm{eV} ; 8.95 ; \mu \mathrm{m} ; 1117 \mathrm{~cm}^{-1}\right)$. The average energy $\left(\Delta \mathrm{E}_{\mathrm{H}} \ldots \mathrm{O}\right)$ of hydrogen $\mathrm{H}$... O-bonds among individual molecules $\mathrm{H}_{2} \mathrm{O}$ after treatment of Drossinakis was measured by NES- and DNES-methods. The result $\Delta \mathrm{E}=-0.0071 \pm 0.0011 \mathrm{eV}$ is according control serum samples of hamsters with cancer and $\Delta \mathrm{E}=-0.0035 \pm 0.0011 \mathrm{eV}$ is for healthy ones. These results suggest the restructuring of $\Delta \mathrm{E}_{\mathrm{H} . . . \mathrm{O}}$ values among $\mathrm{H}_{2} \mathrm{O}$ molecules with a statistically reliable increase of local extremums in DNESspectra. Local extremum was obtained at $(-0.1212) \mathrm{eV}$, which is an indicator of anti-inflammatory effect. Another local extremum was obtained at $(-0.1387) \mathrm{eV}$, which is an indicator of the tumor-development inhibition at the cellular- and the molecular level. The beneficial effects of Drossinakis are characterized as the anti-inflammatory, anti-oxidant, anti-cancer, anti-stroke,etc. This article deals with the review of the basic biophysical-, biochemical- and biological processes - bio-influenced of Drossinakis. In this sense, the experimental research of Toshkova and co-authors has shown increased life span of the tumor-bearing hamsters, bio-influenced by Drossinakis. There are new analyses from hematological studies from Zvetkova.
\end{abstract}

Keywords: Christos Drossinakis - bioinfluence, Infrared thermal field (ITF), Electromagnetic fields, Experimental myeloid tumor of Graffi, hematological studies - erythrocytes

DOI: $10.7176 / \mathrm{JMPB} / 54-02$

Publication date: April $30^{\text {th }} 2019$

\section{Introduction}

The strong bio-influence of Drossinakis has been proved via the application of the methods biophotons (Popp), thermovision (Schlebusch), spectrum analysis of water (Antonov, Ignatov), color coronal spectral analysis (Ignatov), gas discharge visualization (Korotkov), synchronizing of brain electromagnetic waves (Li Gendinovich) etc. at more than 80 institutes and centers in Europe. Drossinakis has achieved the following results according the norm - biophoton emission (Popp) ( 900 biophotons $1 \mathrm{~cm}^{2} / 1 \mathrm{~s}$ ), (norm $\sim 85$ photones, 1 $\mathrm{cm}^{2} / 1 \mathrm{~s}$ ); increasing of the temperature after bioinfluence (Schlebusch) $\left(1.6-1.8^{\circ} \mathrm{C}\right.$ ) (norm $0.1^{\circ} \mathrm{C}$ ); average energy of hydrogen bonds among water molecules according control sample (Antonov, Ignatov) $( \pm 8.2 \mathrm{meV})$ $( \pm 1.1 \mathrm{meV})$ etc.

In the current study was followed the effect of influence with Infrared thermal field (ITF) and electromagnetic fields (e.m. fields) of Christos Drossinakis over the experimental myeloid tumor of Graffi, implanted in hamsters. The study was conducted by a team under the authority of Reneta Toshkova. There are new analyses from hematological studies of Elissaveta Zvetkova.

Purpose of the research: To establish the therapeutic effect of infrared thermal field and e.m. fields in hamsters 
with experimental tumor.

\section{Methods}

\subsection{Experimental animals}

Hamsters, breed "Golden Syrian", aged 2-4 months with weight around 90-100 g, delivered from breeding organization - Slivnitza - Bulgarian Academy of Sciences (BAS) were used in the trials. The animals were grown in standard conditions in the vivarium of the Institute of experimental morphology, pathology and anthropology with museum (IEMPAM) - BAS, in individual plastic cages with free access to food and water.

\subsection{Experimental tumor model}

The experimental Graffi solid tumor was supported once monthly in hamsters from the research team at IEMPAM-BAS (Toshkova, 1995) via subcutaneous transplantation of tumor cells $\left(1-2.10^{6}\right)$ in the back. Between days 7 and 15 after the injection of tumor cells tumor appears, grows progressively and the hamsters die around 30-35 days. In this tumor model, $100 \%$ tumor transplantation and 100\% mortality were observed. No spontaneous tumor's regression was observed.

\subsection{Hematology examination}

Blood smears from Graffi tumour-bearing /control-/ hamsters as well as from the experimental animals /bioinfluenced simultaneously with tumors' implantation/, have been prepared, stained by May-Gruenwald Giemsa method and examined light-microscopically.

\subsection{Ethical aspects:}

All experiments were conducted in accordance with the European convention for protection of vertebrate animals, used for experimental and other scientific purposes (OJ L 222) and approved from the National Veterinary Medical Office.

\section{Results of Hematological studies}

Differences in the erythrocyte /RBC-/ morphology and degree of red cell differentiation were evaluated in the blood smears of controls /healthy hamsters and untreated-tumor-bearing animals/, vs. bio-influenced tumorbearing hamsters - with implanted myeloid tumours of Graffi /as shown in Fig. 1 - A, B, C/.

The data obtained probably indicates a positive bio-influence of Infrared Thermal Biofield (ITF) and electromagnetic fields (e.m. fields) on the erythrocyte (RBC-) membranes' biochemistry, structure and functions (Komorowska, Czyzewska, 1997; Walski et al., 2015) and/or on the erythropoiesis in the Graffi myeloid tumour-bearing hamsters. The authors obtained that the erythrocyte exposure to ITF and e.m. fields leads to structural changes in the phosphor-lipid areas of red cell membranes - strongly influencing the structure and functions of the lipid bilayers. After ITF and e.m. fields exposure RBC are also protected against oxidative stress. By this way, the ITF and e.m. fields effects could lead to the improvement of the anemia-syndrome obligatory presented during the development of Graffi myeloid tumors, as well as in other experimental models of malignancies. In this sense ITF and e.m. fields exerts beneficial effects on cells- in the treatment of cancer, strokes and neurodegenerative disorders including. (Walski et al., 2015). After the same authors, the biomechanisms of the ITF and e.m. fields action is probably of photochemical nature, however it is not fully understood. However, further experimental studies are required for creation of definitive conclusions.

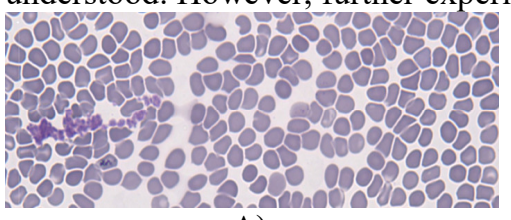

A)

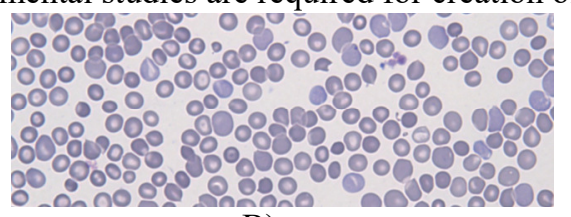

B)

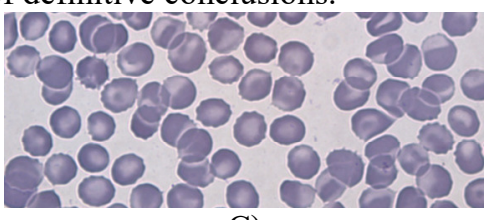

C)

Fig. 1 . Light-microscopic characteristics of erythrocytes (RBCs) in the blood smears /stained by the method of May-Grunwald-Giemsa/:

- control groups \{A - healthy animals (x 20); B - Graffi myeloid tumors-bearing hamsters (x 20)\};

- experimental group $\{\mathrm{C}$ - bio-influenced tumor-bearing hamsters - with implanted myeloid tumors of Graffi $(x 40)\}$. One could see normal erythrocytes (discocytes) (A), anizocytosis and poikilocytosis of RBCs, as biomsarker of anemia - in the blood smears of untreated, tumor-bearing animals (B), as well as RBCs transformed into discocytes, stomatocytes and echinocytes - in the tumor-bearing animals, bioinfluenced with ITF and e.m. fields $(\mathrm{C})$.

\section{Conclusions}

In conclusion, the results achieved from the two types of tests with 5 days course of ITF and e.m. waves 
treatment of hamsters with experimental subcutaneous tumor are positive. Prolonged survival rate and decreased mortality (in the first and second trial), as well as lowered transplantability and slowed tumor growth (in the second trial) were observed. The present results are the base for conducting further tests that aim to establish the optimum regimen of bioinfluence with regards to frequency and duration of the therapeutic procedures, also valid with other experimental models.

The basic conclusion is that Drossinakis is able to increase the average energy of hydrogen bonds among water molecules in the blood of hamsters with cancer after treatment compared to the average energy of hydrogen bonds among water molecules in the blood of non-treated hamsters with cancer and healthy hamsters as control groups. The mathematical model of blood serum solution of hamsters with cancer after the Drossinakis' influence gives significant information about the possible number of hydrogen bonds as a percent of water molecules with different distribution of energy relative to the same number in the two control groups. (Ignatov, Toshkova, Gluhchev, Drossinaksi, 2019)

As a result of different energies of hydrogen bonds, the surface tension of the blood serum solution of cancer hamsters is increased after the treatment relative to the control samples. This effect is connected with the preservation and increase in the energy of the biochemical processes between water molecules and biomolecules.

The achieved results of hamsters from experimental bio-influence of Christos Drossinakis reveal their biological efficiency and can be subject of future studies. Extending the life of the hamsters is an indicator of improving immune status. The results obtained also correspond to data from the medical scientific literature on the positive effects of the near infrared irradiation on the structure and function of erythrocyte membranes - in normal and pathological conditions: after ITF and e.m. fields exposure erythrocytes could be protected against oxidative stress.

The mitochondrial polarity in cancer cells was found to be lower than that of the normal cells: Drossinakis is increasing the mitochondrial polarity.

\section{References}

Adelman, R., Saul, R. \& Ames, B. (1988) Oxidative Damage to DNA: Relation to Species Metabolic Rate and Life Span, $P N A S$, 85(8): 2706-2708.

Anderson, L.E. (1993) Biological Effect of Extremely Low Frequency Electromagnetic Fields: in vivo Studies. Am. Ind. Hig. Assoc. J., 54: 186-196.

Antonov, A. Galabova, T. (1992) Ext. Abstr. Of the $6^{\text {th }}$ National Conference of Biomedical Physics and Engeneering, 60.

Antonov, A., Galabova, T., Todorova, L., Tomov, (1993) Observatoire de Montagne de Mussala OM2, Edit par J.P. Carbonel and J. N. Stamenov, Sofia, 113.

Barnes, F.S. \& Greenebaum, B. (eds.) (2006) CRC Handbook on Biological Effects of Electromagnetic Fields, 3d Edition, Boca Raton: CRC Press, 2, 1-960.

Brubach, J.B., Mermet, A., Filabozzi, A., Gerschel, A. \& Roy, P. (2005) Signatures of the Hydrogen Bonding in the Infrared Bands of Water. J. Chem. Phys., 122: 184509.

Burger, O., Baudish, A. \&Vaupel, J. W. (2012) Human Mortality Improvement in Evolutionary Context, PNAS, 109(44): 18210-18214.

Cleary, S.F. (1993) A Review of in vitro Studies: Low-frequency Electromagnetic Fields. J. Am. Ind. Hyg. Assoc, 54(4): 178-185.

Chang, J.J., Fisch, J. \& Popp, F.A. (eds) (1998) Biophotons. Dordrecht, Kluwer Academic Publishers, 1-417

Cohen, S. \& Popp, F.A. (1997) Biophoton Emission of the Human Body. Journal of Photochemistry and Photobiology B: Biology, 40(2): 187-189.

Drossinakis, Ch., Toshkova, R., Zvetkova, E.\&Gluhchev, G. (2019) Methods of Research in Vivo Research of Therapeutical Effect in Hamsters with Experimental Myeloid Tumor of Graffi, 8th World Congress on Immunology, Pulsus, London, 3: 21.

Edwards, R. et al. (1989) Light emission from the Human Body. Complement Med. Res., 3:16.

Eisenberg, D. \& Kauzmann, W. (1969) The Structure and Properties of Water. Oxford University Press, London. Frey, A.H. (1993) Electromagnetic Field Interactions with Biological Systems. FASEB Journal, 7(2): 272-281

Gluhchev, G., Ignatov, I. Karadzhov, S., Miloshev, G., Ivanov, N.\& Mosin, O.V. (2015) Electrochemically Activited Water. Biophysical and Biological Effects of Anolyte and Catholyte as Types of Water, Journal of Medicine, Physiology and Biophysics, 10: 1-17.

Goryainov S.V. (2012) A Model of Phase Transitions in Double-well Morse Potential: Application to Hydrogen Bond. Physica B, 407, 4233-4237.

Gulyaev, Yu.V. \& Godik, E.E. (1984) On the Possibilities of the Functional Diagnostics of the Biological Subjects Via Their Temporal Dynamics of the Infrared Images, USSR Academy Nauk Proceedings/Biophysics, 277: 1486-1491.

Gulyaev, Yu.V. \& Godik, E.E. (1990) Human and Animal Physical Fields. Scientific American, 5: $74-83$. 
Gulyaev, Yu.V. \& Godik, E.E. (1991) Functional Imaging of the Human Body. IEEE Engineering in Medicine and Biology, 10: 21-29.

Holzel, R. \& Lamprecht, I. (1994) Wirkungen Elektromagnetischer Felder auf Biologische Systeme, Nachrichtentech Elektron, 44(2): 28-32.

Howard, C. \& Hopps (1986) Chemical Qualities of Water that Contribute to Human Health in a Positive Way, Science of the Total Environment, 54: 207-216.

Ignatov, I., Mosin, O. V. \& Naneva, K. (2012) Water in the Human Body is Information Bearer about Longevity. Euromedica, Hanover: 110-111.

Ignatov, I., Antonov, A.\&Galabova, T. (1998) Medical Biophysics - Biophysical Fields of Man, Gea Libris, 171.

Ignatov, I. (2007) Energy Biomedicine, Origin of Living Matter, "Informationability" of Water, Biophysical Fields, ICH, Munich, 1-136

Ignatov I. (2012) Conference on the Physics, Chemistry and Biology of Water, Water in the Human Body is Information Bearer about Longevity, NY, Vermont Photonics.

Ignatov, I. \& Mosin, O.V. (2013) Structural Mathematical Models Describing Water Clusters. Journal of Mathematical Theory and Modeling, 3(11): 72-87.

Ignatov, I. \& Mosin, O. V. (2014) Nature of Haydrogen Bonds in Liquids and Crystals. Ice Crystal Modifications and Their Physical Characteristics, Journal of Medicine, Physiology and Biophysics, 4: 58-80.

Ignatov, I., Mosin, O.V., Velikov, B., Bauer, E. \& Tyminski, G. (2014) Research of Longevity Factors and Mountain Water as a Factor in Teteven, Yablanitsa and Ugarchin Municipalities, Lovech Region, Bulgaria, Journal of Health, Medicine and Nursing, 4: 21-36.

Ignatov, I. \& Mosin, O. V. (2014) Hydrogen Bonds among Molecules in Liquid and Solid State of Water. Modifications of Ice Crystals, Journal of Health, Medicine and Nursing, 5: 56-79.

Ignatov, I. \& Mosin, O.V. (2014b) Methods for Measurements of Water Spectrum. Differential Non-equilibrium Energy Spectrum Method (DNES), Journal of Health, Medicine and Nursing 6: 50-72.

Ignatov, I. \& Mosin, O.V. (2015) Methods for Research of Mountain and Melt Water as Factor of Longevity. Chemical Composition, NES and DNES Methods for Spectral Analysis. Effects of Calcium, Magnesium, Zinc and Manganese, Advances in Physics Theories and Applications, 44: 48-64.

Ignatov, I. \& Mosin, O.V. (2015b) Water: Solid and Liquid Phases. Nano Structures in the Water in Solid and Liquid Phases, Journal of Medicine, Physiology and Biophysics, 9: 82-109.

Ignatov, I.\&Mosin,O.V. (2015) Nanostructures in Solid and Liquid Phases of Water, Journal of Medicine, Physiology and Biophysics, 12:31-57.

Ignatov, I.\&Mosin, O. V. (2014) Isotopic Composition of Water as Main Factor for Longevity, Drug Development and Registration, 9 (4): 146-155.

Ignatov, I. (2018) Research of the Factors of Health and Longevity for the Population in Bulgaria, Bulgarian Journal of Public Health, 10 (3): 52-85.

Ignatov, I., Karadzhov, S., Gluhchev, G.\&Yakimov, I. (2018) Electromagnetically Activated Water - Properties and Effects, Bulgarian Journal of Public Health, 10 (4): 63-69.

Ignatov, I., Toshkova, R., Gluhchev, G.\&Drossinakis, Ch. (2019) Results of Blood Serum from Cancer Treated Hamsters with Infrared Thermal Field and Electromagnetic Fields, Journal of Health, Medicine and Nursing, 58: 101-112.

Isaacs E.D., Shukla A., Platzman P.M, et all. (2000) Compton Scattering Evidence for Covalency of the Hydrogen Bond in Ice, J. Phys. Chem. Solids, 61, 403-406.

Keutsch F. \& Saykally R. (2011) Water Clusters: Untangling the Mysteries of the Liquid, One Molecule at a Time. PNAS, 98(19), 105330-10540.

Komorowska M., Czyzewska H. (1997) ). The effect of near infrared radiation on erythrocyte membranes; EPR study. Nukleonika. 42/2

Krasnov, V.V. \& Gordetsov, A.S. (2009) Infrared spectral analysis of blood serum as level of disturbances of metabolic processes in infusion children pathology. Clinical Medicine: 83-94 [in Russian].

Kwan-Hoong, Ng. (2003) Non-ionizing Radiations - Sources, Biological Effects, Emissions and Exposures. Proceedings of the International Conference on Non-Ionizing Radiation at UNITEN (ICNIR2003). Electromagnetic Fields and Our Health. 20-22 October 2003.

Luck W., Schiöberg D. \& Ulrich S. (1980) Infared Iinvestigation of Water Structure in Desalination Membranes. J. Chem. Soc. Faraday Trans., 2(76), 136-147.

Niggli, H. (1993). Artificial Sunlight Irradiation Induces Ultra Weak Photon Emission in Human Skin Fibroblasts, Journal of Photochemistry and Photobiology B: Biology, 18 (2-3): 281-285.

Mariani, E. et al. (2006) Antioxidant Enzyme Activities in Healthy Old Subjects: Influence of Age, Gender and Zinc Status: Results from the Zincage Project, Biogerentology, 7 (5-6): 391:398.

Marino, A.A (Ed.) (1988) Modern Bioelectricity. Marieel Dekker, New York, Basel, ISBN 0-8247-7788-3. 
Marinov, M. \& Ignatov, I. (2008) Color Kirlian Spectral Analysis. Color Observation with Visual Analyzer. Euromedica, Hanover, 57-59.

Miller, M.W. (1986) Extremely Low Frequency (ELF) Electric Fields: Experimental Work on Biological Effects. CRC Handbook of Biological Effects of Electromagnetic Fields, 138-168.

Malhotra, A.,\&Dhawan, D. K. (2008) Zinc Improves Antioxidative Enzymes in Red Blood Cells and Hematology in Lithium-Treated Rats, Nutr. Res., 28(1):43-50

Pasichnyk I. Everaers R. \& Maggs A.C. (2008) Simulating van der Waals-interactions in Water/HydrocarbonBased Complex Fluids. J. Phys. Chem. B, 112(6), 1761-1764.

Pauling L. (ed.) (1960) The Nature of the Chemical Bond and the Structure of Molecules and Crystals, New York: Cornell University Press.

Pocock, S.J, Shaper, A.G. \& Packham, R.F. (1981) Studies of Water Quality and Cardiovascular Disease in the United Kingdom, Sci. Total Environ., 18: 25-34.

Toshkova, R., Ignatov, I., Zvetkova, E., Gluhchev, G. (2019) Bioinfluence with Infrared Thermal and Electromagnetic Fields as a Therapeutic Approach of Hamsters with Experimental Graffi Myeloid Tumor, Journal of Natural Sciences Research, 9 (4):1-11.

Walski T., Dyrda A., Dzik M., et al. (2015) Near infrared light induces post-translational modifications of red blood cell proteins. Photochemical and Photobiological Sciences, 14 (11). 